\title{
Sparse reconstruction of compressive
} sensing MRI using cross-domain stochastically fully connected conditional random fields

\author{
Edward Li ${ }^{1}$, Farzad Khalvati ${ }^{2}$, Mohammad Javad Shafiee ${ }^{1}$, Masoom A. Haider ${ }^{2}$ and Alexander Wong ${ }^{1 *}$
}

\begin{abstract}
Background: Magnetic Resonance Imaging (MRI) is a crucial medical imaging technology for the screening and diagnosis of frequently occurring cancers. However, image quality may suffer from long acquisition times for MRIs due to patient motion, which also leads to patient discomfort. Reducing MRI acquisition times can reduce patient discomfort leading to reduced motion artifacts from the acquisition process. Compressive sensing strategies applied to MRI have been demonstrated to be effective in decreasing acquisition times significantly by sparsely sampling the $k$-space during the acquisition process. However, such a strategy requires advanced reconstruction algorithms to produce high quality and reliable images from compressive sensing MRI.
\end{abstract}

Methods: This paper proposes a new reconstruction approach based on cross-domain stochastically fully connected conditional random fields (CD-SFCRF) for compressive sensing MRI. The CD-SFCRF introduces constraints in both $k$-space and spatial domains within a stochastically fully connected graphical model to produce improved MRI reconstruction.

Results: Experimental results using T2-weighted (T2w) imaging and diffusion-weighted imaging (DWI) of the prostate show strong performance in preserving fine details and tissue structures in the reconstructed images when compared to other tested methods even at low sampling rates.

Conclusions: The ability to better utilize a limited amount of information to reconstruct T2 $w$ and DWI images in a short amount of time while preserving the important details in the images demonstrates the potential of the proposed CD-SFCRF framework as a viable reconstruction algorithm for compressive sensing MRI.

Keywords: Compressive sensing, Conditional random fields, Magnetic resonance imaging

Abbreviations: MRI, Magnetic resonance imaging; CRF, Conditional random field; CD-SFCRF, Cross-domain stochastically fully connected conditional random fields; T2W, T2-weighted; DWI, Diffusion-weighted imaging; MP-MRI, Multi-parametric MRI; CDI, Correlated diffusion imaging; TV, Total variation; ADC, Apparent diffusion coefficient; PSNR, Peak signal-to-noise ratio; stdev, Standard deviation; CAD, Computer-aided diagnosis; CD-MRI, Computational diffusion MRI

\footnotetext{
*Correspondence: a28wong@uwaterloo.ca

${ }^{1}$ Department of Systems Design Engineering, University of Waterloo, Waterloo, Ontario, Canada

Full list of author information is available at the end of the article
} 


\section{Introduction}

Magnetic Resonance Imaging (MRI) is a medical imaging technology that is currently used for diagnostic imaging of a wide range of diseases. In particular, since MRI does not use ionizing radiation, it has become a crucial imaging modality for screening frequently occurring cancers such as prostate cancer in men, breast cancer in women, as well as lung and colorectal cancer for both men and women. In 2015, 196,900 new cases of cancer (excluding non-melanoma skin cancers) were expected, with $51 \%$ of these belonging to the four aforementioned types of cancer in Canada [1]. As such, cancer screening methods with accurate and reliable information such as MRI is highly desired. Of particular interest for cancer screening is multi-parametric MRI (MP-MRI) since more information can be acquired through different modalities. MP-MRI contains different techniques such as diffusion weighted imaging (DWI), correlated diffusion imaging (CDI) [2-4], dynamic contrast enhancement (DCE), T2-weighted (T2w) imaging, and T1-weighted (T1w) imaging [5]. Although this approach provides a more complete information, acquisition times are significantly longer which causes more patient discomfort and motion artifacts that decrease image quality. As a result, new methods to improve MRI acquisition times are highly desired to facilitate for reliable MP-MRI data acquisition.

Compressive sensing has been demonstrated to be an effective strategy for reducing MRI acquisition times by acquiring significantly fewer samples in $k$-space. A complete signal can then be fully reconstructed through sparse, yet sufficient number of samples [6-8]. In MRI, compressive sampling strategies have been demonstrated to be highly effective at reducing acquisition time while maintaining image quality as different types of tissue structure have been shown to be sparse in certain domains [9]. Furthermore, different techniques have been proposed to improve the imaging process [10] as well as the reconstruction process [11-23] in compressive sensing. Due to the limited amount of data available through compressive sensing, advanced reconstruction algorithms are required to produce high quality and reliable images.

Different methods have been proposed for sparse reconstruction of compressive sensing MRI [11-23]. As a notable example, Block [14] proposed an iterative image reconstruction technique using a modified total variation (TV) constraint $[20,21]$ for sparse reconstruction of compressive sensing brain MRI. Trzasko [15] introduced a homotopic $l_{0}$ minimization method for the sparse reconstruction of compressive sensing spinal MRI. Wong [12] extended upon this idea and proposed a regional sparsified domain for the sparse reconstruction of breast MRI. A similar technique was also demonstrated by $\mathrm{Qu}$ using combined sparsifying transforms and smoothed $l_{0}$ norm minimization [13], where they showed that the use of combined transforms can improve image quality comprised of the reconstructed images from compressive sensing MRI when compared to methods using a single sparsifying transform. However, the downside of the $l_{0}$ norm minimization is the fact that its performance significantly depends on the tuning parameters where these tuning parameters can greatly affect the convergence rate of the algorithm. Other $l_{n}$ optimization techniques such as the standard $l_{2}$ (least squares) minimization can have high error rates as reported in [24].

An area that is little explored but can reap significant potential benefits is the application of random field modeling for improved sparse reconstruction of compressive sensing MRI. Random field modeling such as Markov random fields (MRF) $[25,26]$ and conditional random fields (CRF) [27] have long been shown to be powerful tools for incorporating spatial context within a probabilistic graphical modeling framework, which can have significant benefits for reconstructing images from sparse measurements. Despite powerful modeling capabilities and potential benefit to sparse reconstruction, one of the biggest hurdles in leveraging random field models for compressive sensing MRI is the fact that all MRI measurements are made in $k$-space, whereas the images are reconstructed in spatial domain. As the majority of random field models are typically modeled in a single domain, such models cannot be used directly for the purpose of sparse reconstruction of compressive sensing MRI. This is further complicated by the fact that the MRI measurements in $k$ space are sparse and incomplete, which make it difficult to leverage existing random field models for this problem. Therefore, a probabilistic graphical modeling framework that can consolidate the fact that partial measurements are made in a domain different than the desired states of the reconstruction images is needed to truly leverage the power of random field modeling for sparse reconstruction of compressed sensing MRI.

This paper proposes a cross-domain Stochastically fully connected conditional random field (CD-SFCRF) approach for the reconstruction of compressive sensing MRI at below Nyquist sampling rates [28]. Inspired by the stochastic cliques method presented in [29], the proposed cross domain model is a significant extension upon this method by consolidating two different domains of spatial domain and $k$-space specifically for MRI compressive sensing. CD-SFCRF framework introduces constraints in both $k$-space and spatial domains within a stochastically fully connected graphical model to produce improved MRI reconstruction. The proposed CD-SFCRF framework has the ability to utilize spatial and data driven consistencies in the spatial domain along with data driven consistencies in the $k$-space domain pertaining to sparse measurements while maintaining edge features and structural details in the reconstructed images. Phantom MRI 
data as well as prostate MRI data captured using T2w and DWI imaging modalities, which also yields apparent diffusion coefficient (ADC) map images, were used to illustrate the efficacy of the proposed CD-SFCRF framework for sparse reconstruction of compressive sensing MRI. To the best of the authors' knowledge, this is the first time that constraints in both $k$-space and spatial domains are used in conjunction within a stochastically fully connected graphical model for the sparse reconstruction of compressive sensing MRI, which is the main contribution of this paper.

The paper is formatted as follows. The methodology behind the proposed CD-SFCRF framework is described in Section "Methodology". The experimental setup is described in Section "Experimental setup". Results and discussions are presented and discussed in Sections "Results" and "Discussion", respectively. Finally, the conclusion is presented in Section "Conclusions".

\section{Methodology}

In MRI, measurements are made in the $k$-space [30], with the lower frequency coefficients in the $k$-space containing coarse-grained contrast information while higher frequency coefficients contain fine-grained image detail information. The MRI measurements from the $k$-space are transformed into the spatial domain to form the reconstructed MRI image. Most compressive sensing strategies $[6,15]$ sparsely sample the $k$-space to reduce image acquisition time significantly. Therefore, to fully utilize available information in the reconstruction process, datadriven constraints in the $k$-space domain and data and spatial driven constraints in the spatial domain would be highly beneficial in improving image reconstruction quality from compressive sensing MRI.

Motivated by this, the proposed cross-domain stochastically fully connected conditional random field (CDSFCRF) introduced here for the purpose of sparse reconstruction of compressive sensing MRI, extends upon the seminal work on stochastically fully connected conditional random fields (SFCRF) first proposed in [29] to facilitate for this cross-domain optimization. SFCRFs are fully-connected conditional random fields with stochastically defined cliques. Unlike traditional conditional random fields (CRF) where nodal interactions are deterministic and restricted to local neighborhoods, each node in the graph representing a SFCRF is connected to every other node in the graph, with the cliques for each node is stochastically determined based on a distribution probability. Therefore, the number of pairwise cliques might not be the same as the number of neighborhood pairs as in the traditional CRF models. By leveraging long-range nodal interactions in a stochastic manner, SFCRFs facilitate for improved detail preservation while maintaining similar computational complexity as CRFs, which makes SFCRFs particularly enticing for the purpose of improved sparse reconstruction of compressive sensing MRI. However, here the problem is to reconstruct an MRI image in the spatial domain while the available measurements are made in $k$-space domain. Similar to most CRF models, SFCRFs cannot be leveraged directly for this purpose. Motivated by the significant potential benefits of using SFCRFs in improving reconstruction quality of compressive sensing MRI, we extend the SFCRF model into a cross-domain stochastically fully connected conditional random field (CD-SFCRF) model that incorporates crossdomain information and constraints from $k$-space and spatial domains to reconstruct the desirable MRI image from sparse observations in $k$-space.

The theory pertaining to sparse reconstruction via a cross-domain stochastically fully connected conditional random field model is detailed in Appendix 1.

\section{Implementation}

An implementation of the proposed CD-SFCRF framework for the purpose of sparse reconstruction from compressive sensing MRI is illustrated in Fig. 1. Here,

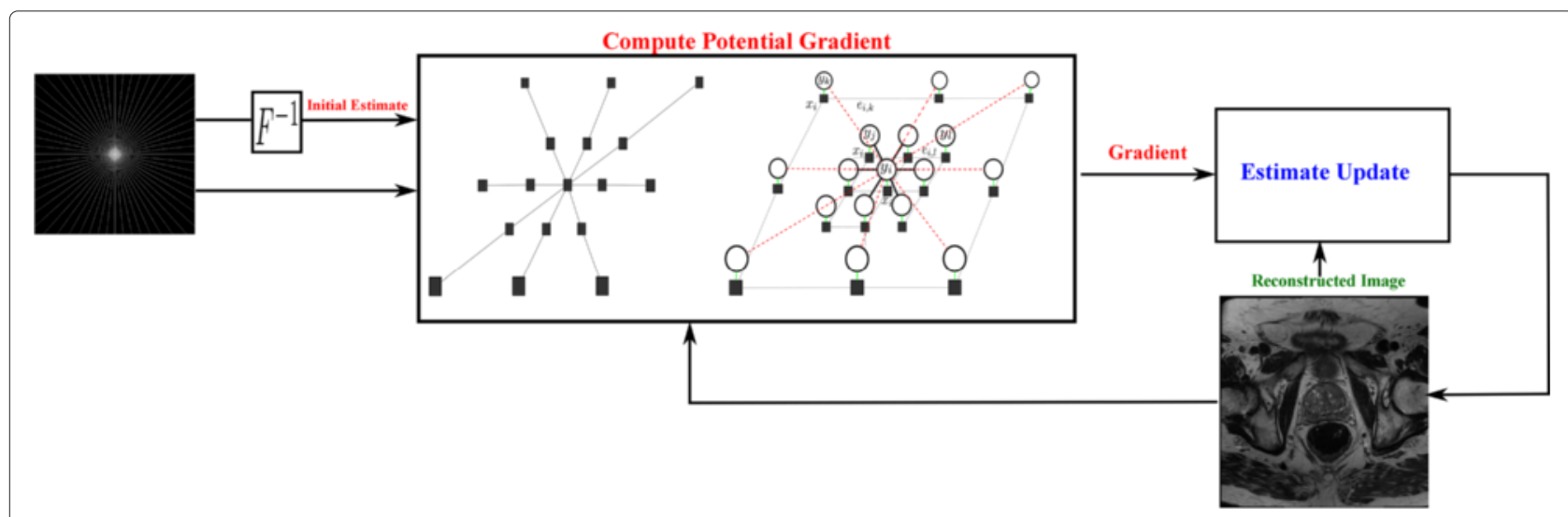

Fig. 1 Optimization framework of the proposed CD-SFCRF framework for sparse reconstruction from compressive sensing MRI 
an iterative gradient descent optimization approach is employed, and can be described as follows. First, the original compressive sensing MRI data in $k$-space is transformed to the spatial domain to provide an initial estimate of the reconstructed image. Second, the gradient of the unary and pairwise energy potentials is computed, where the unary data driven consistencies with respect to the original observations are enforced in the $k$-space, and spatial and data driven consistencies are enforced in the spatial domain. Third, the estimate of the reconstructed image is updated based on the previous estimate and the computed gradient. The second and third steps of this process are repeated until convergence.

\section{Experimental setup}

To study the efficacy of the proposed CD-SFCRF method for the purpose of sparse reconstruction of compressive sensing MRI, experiments were performed including: i) MRI data acquired of a MRI training phantom, and ii) prostate MP-MRI data of 20 patient cases. A detailed description of the phantom data, patient data, and MRI image acquisition procedure to facilitate for the various experiments are described below.

\section{Phantom data}

The MRI training phantom used in the experiments, shown in Fig. 2, was a multi-modality prostate training phantom from Computerized Imaging Reference Systems Inc (CIRCS MODEL 053). The phantom is composed of a clear acrylic container with dimensions $11.5 \times 7.0 \times 9.5 \mathrm{~cm}$ with a front probe opening of $3.2 \mathrm{~cm}$ diameter and a rear

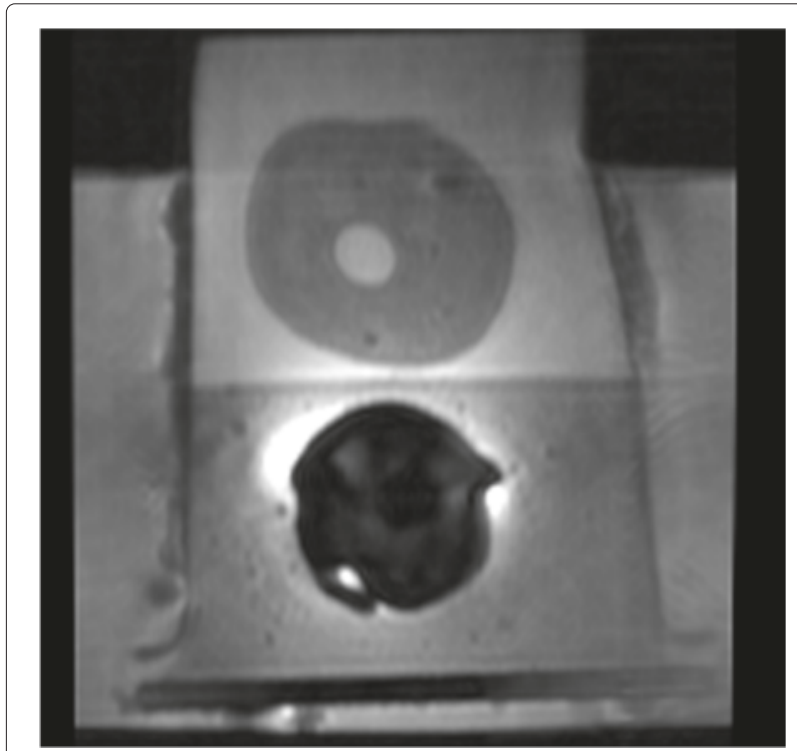

Fig. 2 Example slice of the prostate training phantom from Computerized Imaging Reference Systems Inc (CIRCS MODEL 053) used for evaluation purposes probe opening of $2.6 \mathrm{~cm}$ diameter. The prostate is composed of high-scattering Blue Zerdine with dimensions $5 \times 4.5 \times 4.0 \mathrm{~cm}$ and is placed in a background gel similar to water with little backscatter attenuation $(\leq 0.07 \mathrm{~dB} / \mathrm{cm}-$ $\mathrm{MHz}$ ). Within the prostate, there are 3 randomly placed lesions of sizes between $0.5-1.0 \mathrm{~cm}$ placed hypoechoic to the prostate. The urethra and rectal wall are made of low scattering Zerdine with diameter of $0.7 \mathrm{~cm}$ with dimensions $6 \times 11 \times 0.5 \mathrm{~cm}$, respectively. This phantom was imaged with an inflatable Medrad eCoil ERC using DWI. The DWI MRI was acquired by a 3T GE Discovery MR750. DWI was collected at $b=0 \mathrm{~mm}^{2} / \mathrm{s}$ at 3-NEX ${ }^{2}$. For the DWI data, the echo time (TE) was $71.70 \mathrm{~ms}$ and repetition time (TR) was $10,000.00 \mathrm{~ms}$.

\section{Patient data experiments}

To test the efficacy of the proposed CD-SFCRF framework within a real clinical scenario, MRI data of 20 patients (17 with cancer and 3 without cancer) were acquired using a Philips Achieva 3.0T machine at Sunnybrook Health Sciences Centre, Toronto, Ontario, Canada. All data was obtained retrospectively under the local institutional research ethics board (Research Ethics Board of Sunnybrook Health Sciences Centre). For each patient, the following MP-MRI modalities were obtained (Table 1): T2w and DWI. The patients' age ranged from 53 to 83 . Table 1 summarizes the information about the 20 patients' datasets used in this study, which includes displayed field of view (DFOV), resolution, echo time (TE), and repetition time (TR).

\section{Compressed sensing configuration}

In order to evaluate the efficacy of the proposed CDSFCRF framework at different sample rates, we first acquired MRI measurements at all $k$-space coefficients. Based on this fully-sampled set of $k$-space measurements, sparse sampling was then conducted using radial sampling patterns with different numbers of radial sampling lines to achieve a desired sampling rate. For example, Fig. 3 shows a radial sampling pattern which corresponds to a sampling rate of $32 \%$ of the $k$-space. Different sampling rates were tested and evaluated in this study.

\section{Results}

In order to evaluate the efficacy of the proposed CD-SFCRF framework for sparse reconstruction of compressive MRI sensing, a comparative evaluation analysis

Table 1 Description of the prostate T2w and DWI images

\begin{tabular}{lllll}
\hline Modality & DFOV $\left(\mathrm{cm}^{2}\right)$ & Resolution $\left(\mathrm{mm}^{3}\right)$ & TE $(\mathrm{ms})$ & TR $(\mathrm{ms})$ \\
\hline T2W & $22 \times 22$ & $0.49 \times 0.49 \times 3$ & 110 & 4,687 \\
DWI & $20 \times 20$ & $1.56 \times 1.56 \times 3$ & 61 & 6,178 \\
\hline
\end{tabular}




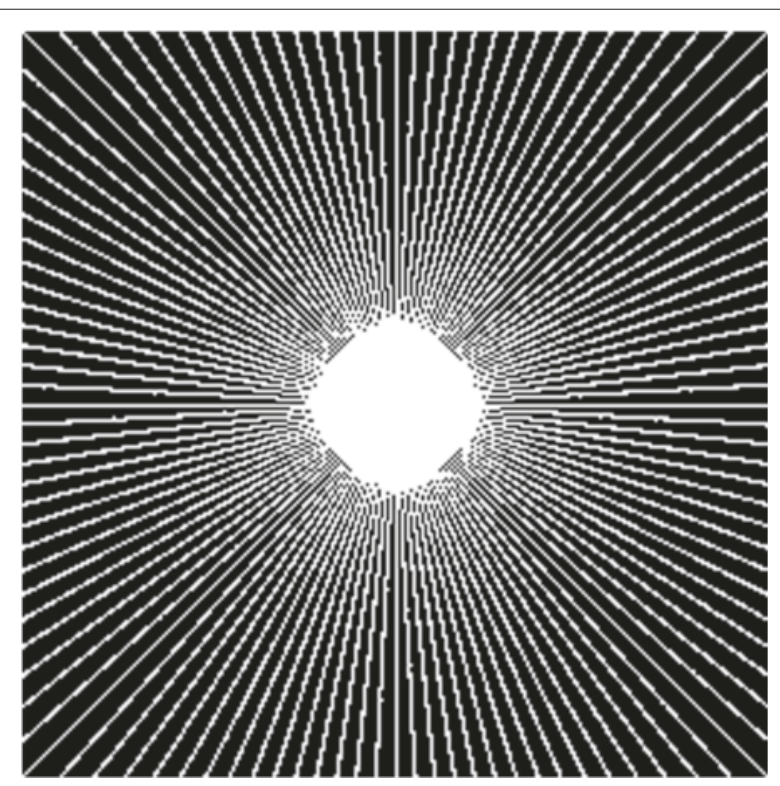

Fig. 3 Radial $k$-space sampling pattern at $32 \%$ sampling rate

was performed alongside a baseline $l_{2}$ minimization $\left(L_{2}\right)$ reconstruction method, and a state-of-the-art homotopic $l_{0}$ minimization $\left(\mathrm{H} L_{0}\right)$ [15] reconstruction method. The tested methods were compared quantitatively through peak signal-to-noise ratio (PSNR) analysis, and qualitatively via visual assessment. All tested methods were implemented based on the original literature, with optimal parameters used in this study. All tested methods were run until convergence.

Figure 4 shows the PSNR versus sampling rate plots for the tested methods for the phantom MRI data.

Tables 2, 3, and 4 show the PSNR results for the three reconstructed methods for the T2w, DWI, as well as ADC map images for the patient data experiments at different sampling rates.

Figures 5 and 6 shows the visual comparison between the reconstructed images produced using the proposed CD-SFCRF framework compared with that produced using the $L_{2}$ and homotopic $l_{0}$ minimization reconstruction methods for three cases for $\mathrm{T} 2 \mathrm{w}$ images.

Figures 7 and 8 shows the visual comparison between the reconstructed images produced using the proposed CD-SFCRF framework compared with that produced using the $L_{2}$ and $\mathrm{H} L_{0}$ methods for three patient cases for DWI $\left(b=100 s / m^{2}\right)$ and ADC maps.

\section{Discussion}

As it can be observed from Fig. 4, the proposed CDSFCRF framework achieved noticeable PSNR improvements over the other tested methods at all tested sampling

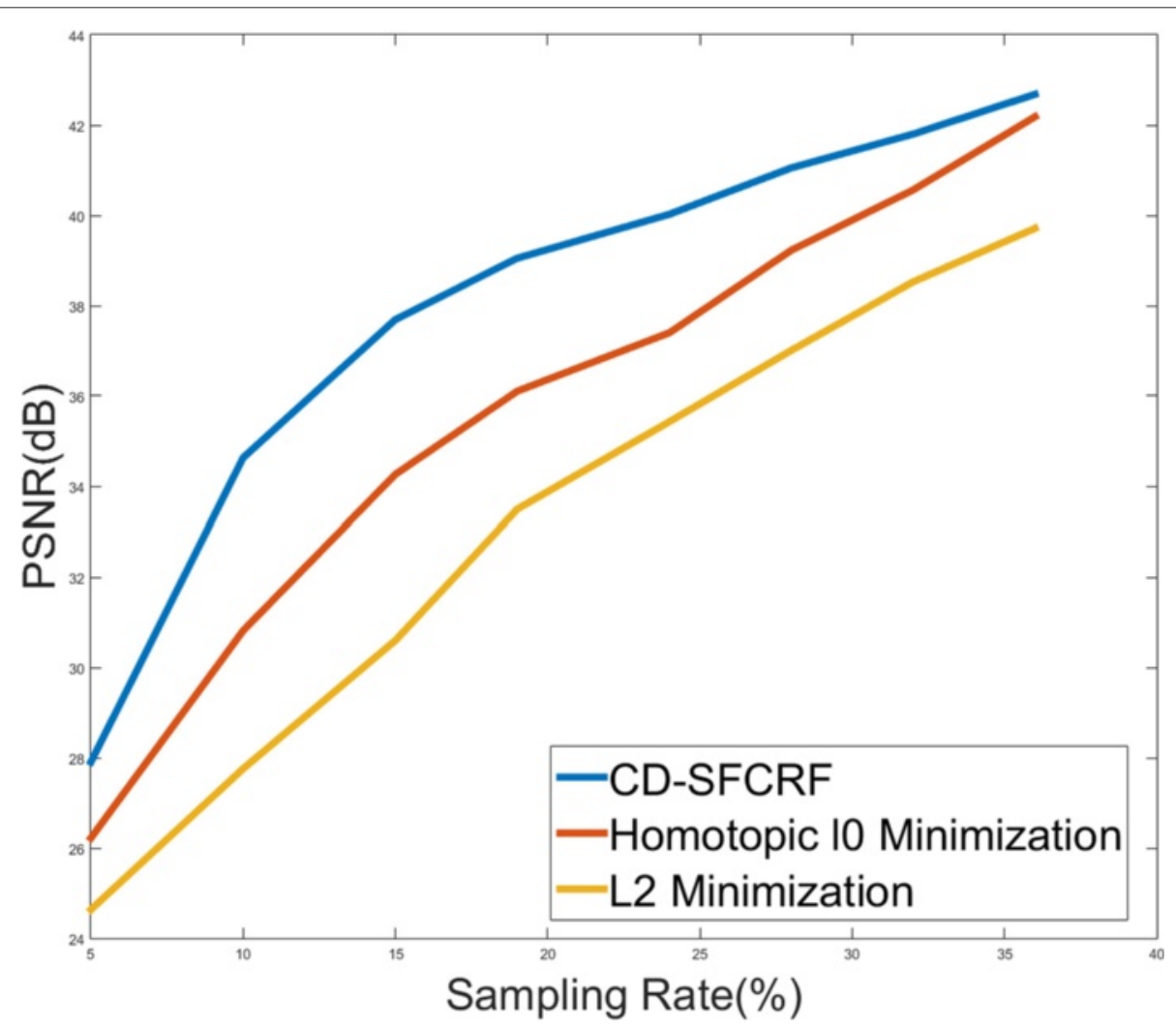

Fig. 4 PSNR vs. sampling rates plots for the tested methods for the phantom MRI data at different sampling rates 
Table 2 PSNR \pm standard deviation (stdev) for T2w images for the patient data experiments (24 images/patient for 20 patients) across different methods with $P$-values comparing $L_{2}$ and $H_{0}$ methods with CD-SFCRF, respectively

\begin{tabular}{llllll}
\hline Sampling rate $(\%)$ & $L_{2}(\mathrm{~dB})$ & $P$-value & $H L_{0}(\mathrm{~dB})$ & $P$-value & CD-SFCRF $(\mathrm{dB})$ \\
\hline 17 & $25.56 \pm 0.92$ & $p \ll 0.0001$ & $26.22 \pm 1.01$ & $p \ll 0.0001$ & $\mathbf{2 7 . 3 4} \pm \mathbf{1 . 1 6}$ \\
32 & $28.39 \pm 0.98$ & $p \ll 0.0001$ & $28.80 \pm 1.01$ & $p \ll 0.0001$ & $\mathbf{2 9 . 7 2} \pm \mathbf{1 . 1 6}$ \\
47 & $30.42 \pm 1.05$ & $p \ll 0.0001$ & $30.80 \pm 1.05$ & $p \ll 0.0001$ & $\mathbf{3 1 . 2 3} \pm \mathbf{1 . 1 7}$ \\
\hline
\end{tabular}

Bold face indicates the method with the highest performance metric

rates. The CD-SFCRF produced improvements of up to $4 d B$ over $\mathrm{H} L_{0}$ and $7 d B$ over $L_{2}$ in low sampling conditions. It can also be observed that as sampling rates increase, the performance differences decrease. This is due to the fact that as the sampling rate increases, the amount of available measurements increases, and as such the level of reconstruction quality improvements that can be achieved will naturally decrease given the amount of available information becomes increasingly sufficient for high quality reconstruction. The ability of the CDSFCRF framework to produce high quality reconstruction at very low sampling rates can be demonstrated visually as well.

From additional quantitative analysis of patients MRI data presented in Tables 2, 3, and 4, it can be observed that the proposed CD-SFCRF framework achieved the greatest PSNR improvements for the lowest sampling rate (i.e., $17 \%$ ) where for T2w, CD-SFCRF improved PSNR by $1.78 \mathrm{~dB}$ and $1.12 \mathrm{~dB}$ over the $L_{2}$ and $\mathrm{H} L_{0}$ methods, respectively. For DWI, CD-SFCRF improved PSNR by $1.85 \mathrm{~dB}$ and $0.28 \mathrm{~B}$ over the $L_{2}$ and $\mathrm{H} L_{0}$ methods, respectively. Interestingly for ADC maps, the best improvements in PSNR were achieved for the highest sampling rate (47\%) where CD-SFCRF improved PSNR by $4.44 d B$ and $0.21 B$ over the $L_{2}$ and $\mathrm{H} L_{0}$ methods, respectively.

Tables 2, 3 and 4 also show the $P$-values calculated by comparing the proposed CD-SFCRF method with $L_{2}$ and $\mathrm{H} L_{0}$ methods, respectively. As it can be seen, P-values show significant difference between CD-SFCRF and the other two methods for T2w and DWI images. For ADC maps, the proposed CD-SFCRF was significantly different than $L_{2}$ method as well. The only comparison that did not show significantly different results was CD-SFCRF compared to $\mathrm{H} L_{0}$ method for ADC maps. This shows that the PSNR improvement for the proposed CD-SFCRF framework was meaningful for the majority of cases when compared to other tested methods.

Comparing the results for phantom MRI data (Fig. 4) and patients MRI data shown in Tables 2, 3 and 4 shows that the proposed CD-SFCRF framework yields higher performance improvement at $10-20 \%$ sampling range for phantom MRI data compared to patients MRI data. The reason for this difference on PSNR improvement is the fact that the morphological and textural properties of the phantom is significantly less complex than that of real patients' prostates, and thus the reconstruction problem is a simpler one for the phantom and as a result, greater PSNR gains were achieved using the proposed method.

Qualitative observations from Figs. 5 and 6 show that the $L_{2}$ method resulted in blurry T2w images as well as noticeable radial artifacts at low sampling rates as expected due to the least squares reconstruction being prone to errors. The $\mathrm{H} L_{0}$ approach performed better than the L2 minimization and was able to noticeably reduce artifacts and provide a higher quality reconstruction. However, in comparison, the CD-SFCRF was able to better restore details and fine tissue structure in the reconstructed image when compared to $\mathrm{H} L_{0}$. This is to be expected as the CD-SFCRF takes advantage of more complete data and spatial driven consistencies in a fully connected nature, thus better modeling the underlying tissue detail and structures.

Furthermore, as it can be seen in Figs. 7 and 8, the $L_{2}$ method resulted in blurry DWI and ADC map images again with noticeable radial artifacts. Although the $\mathrm{H} L_{0}$ approach performed better than the $L_{2}$ method, it can be observed once again that the proposed CD-SFCRF approach was able to preserve more fine tissue structure and detail in the reconstructed image when compared to the $\mathrm{H} L_{0}$ method. Nevertheless, an inherent trade-off

Table 3 PSNR \pm stdev for DWI images for the patient data experiments (24 images/b-value for 4 b-values/patient for 20 patients) across different methods with $P$-values comparing $L_{2}$ and $H L_{0}$ methods with CD-SFCRF, respectively

\begin{tabular}{llllll}
\hline Sampling rate $(\%)$ & $L_{2}(\mathrm{~dB})$ & $P$-value & $H L_{0}(\mathrm{~dB})$ & $P$-value & CD-SFCRF $(\mathrm{dB})$ \\
\hline 17 & $26.90 \pm 1.86$ & $p \ll 0.0001$ & $28.46 \pm 2.50$ & $p \ll 0.0001$ & $\mathbf{2 8 . 7 5} \pm \mathbf{2 . 2 2}$ \\
32 & $31.92 \pm 2.32$ & $p \ll 0.0001$ & $33.39 \pm 3.03$ & $p \ll 0.0001$ & $\mathbf{3 3 . 6 1} \pm \mathbf{2 . 1 7}$ \\
47 & $36.45 \pm 2.67$ & $p \ll 0.0001$ & $37.85 \pm 2.48$ & $p \ll 0.0001$ & $\mathbf{3 7 . 9 9} \pm \mathbf{2 . 1 1}$ \\
\hline
\end{tabular}

Bold face indicates the method with the highest performance metric 
Table 4 PSNR \pm stdev for ADC images for the patient data experiments (24 images/patient for 20 patient) across different methods with $P$-values comparing $L_{2}$ and $H L_{0}$ methods with CD-SFCRF, respectively

\begin{tabular}{|c|c|c|c|c|c|}
\hline Sampling rate (\%) & $L_{2}(\mathrm{~dB})$ & $P$-value & $H L_{0}(\mathrm{~dB})$ & $P$-value & CD-SFCRF $(\mathrm{dB})$ \\
\hline 17 & $17.20 \pm 0.61$ & $p \ll 0.0001$ & $19.35 \pm 0.58$ & 0.88 & $19.50 \pm 0.57$ \\
\hline 32 & $18.05 \pm 0.48$ & $p \ll 0.0001$ & $21.66 \pm 0.54$ & 0.89 & $21.72 \pm 0.55$ \\
\hline 47 & $18.72 \pm 0.32$ & $p \ll 0.0001$ & $22.94 \pm 0.39$ & 0.89 & $23.16 \pm 0.37$ \\
\hline
\end{tabular}

Bold face indicates the method with the highest performance metric

exists between preserving fine textural granularity and reducing artifacts due to compressed sensing which can be well utilized in the proposed CD-SFCRF framework to achieve a balance between the two competing constraints.

In Figs. 5, 6, 7 and 8, the tumourous regions marked by a radiologist and confirmed by pathology report (biopsy results) are shown by red arrow or white boundary. It can be seen that the proposed CD-SFCRF method preserves the separability of the cancerous and healthy tissue in all cases, which is an important measure for usability of the proposed method in practice. As it can be seen, the tumourous regions are blurred in the $L_{2}$ method, which may make it difficult to detect for radiologists.

Both quantitative and qualitative analysis demonstrate the potential of the proposed CD-SFCRF framework as a reliable reconstruction approach for compressive sensing in MRI. It demonstrates the ability to produce edge and tissue details at very low sampling rates. The CDSFCRF framework better utilize available information to produce high quality reconstructed images given very limited available information. Preservation of tissue structure and detail enhancement, and noise and artifact mitigation are very important for MRI as the diagnostic quality is directly related to the image quality. This demonstrates that the CD-SFCRF framework can be a viable clinical technique as the reduction in acquisition can lead to faster acquisitions and lower patient wait times. With a lower acquisition time and hence lower patient wait time, patients can have access to the necessary treatments in a timely manner, significantly improving the patient outcome and survival rates.

The compressive sensing method used to reconstruct MR images can influence the performance of the computer-aided diagnosis (CAD) tools. For example, several radiomics-based CAD algorithms have been proposed for automatic prostate cancer detection which use T2w and DWI to extract texture and morphological features fed into a classifier [31-36]. These algorithms

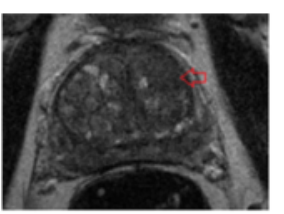

(a) Patient 1 Fully Sampled

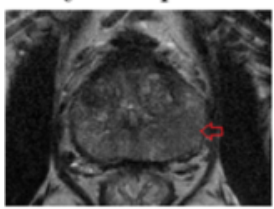

(e) Patient 2 Fully Sampled

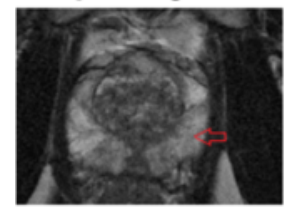

(i) Patient 3 Fully Sampled

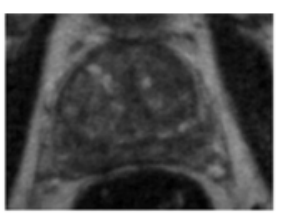

(b) Patient $1 L_{2}$

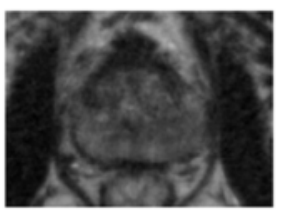

(f) Patient $2 L_{2}$

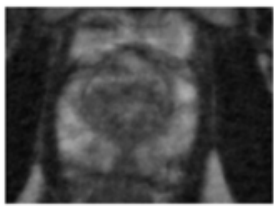

(j) Patient $3 L_{2}$

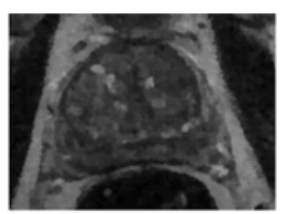

(c) Patient $1 \mathrm{H} L_{0}$

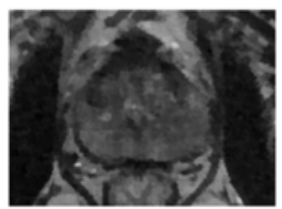

(g) Patient $2 \mathrm{H} L_{0}$

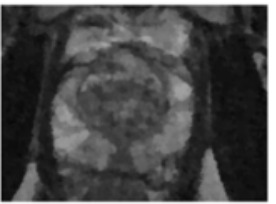

(k) Patient 3 $\mathrm{H} L_{0}$

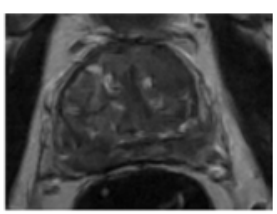

(d) Patient 1 CDSFCRF

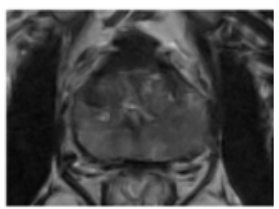

(h) Patient 2 CDSFCRF

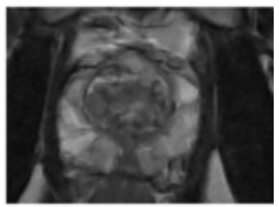

(1) Patient 3 CDSFCRF

Fig. 5 Sample T2w results for 3 patient images produced using CD-SFCRF, $L_{2}$, and $H L_{0}$ at $32 \%$ sampling ratio. Compared to other methods, CD-SFCRF preserves tissue details and contrast especially in the tumourous regions. The arrow shows tumourous region in the fully sampled image (a, e, i) 


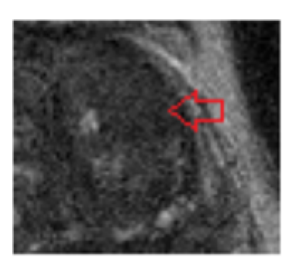

(a) Patient 1 Fully Sampled

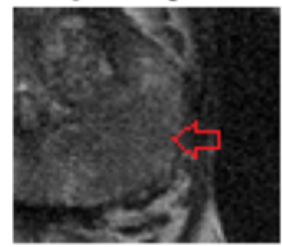

(e) Patient 2 Fully Sampled

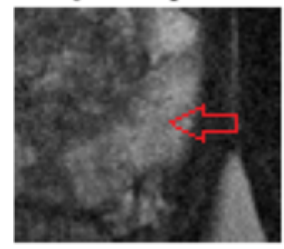

(i) Patient 3 Fully Sampled

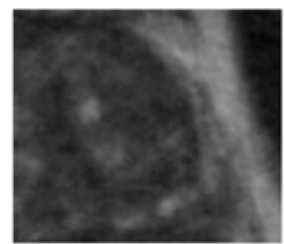

(b) Patient $1 L_{2}$

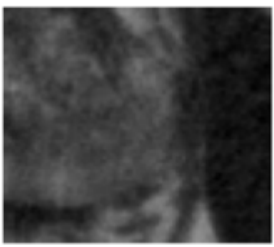

(f) Patient $2 L_{2}$

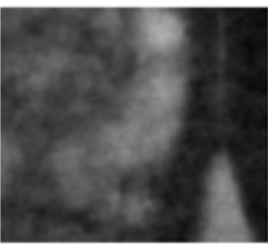

(j) Patient $3 L_{2}$

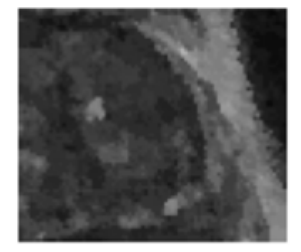

(c) Patient $1 \mathrm{H} L_{0}$

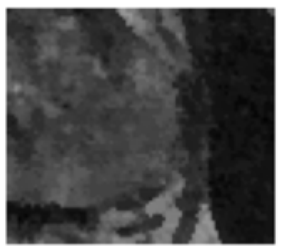

(g) Patient $2 \mathrm{H} L_{0}$

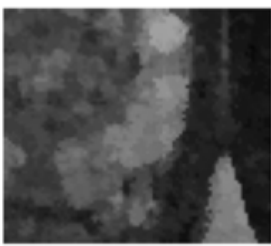

(k) Patient 3

$\mathrm{H} L_{0}$

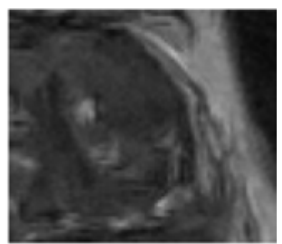

(d) Patient 1 CDSFCRF

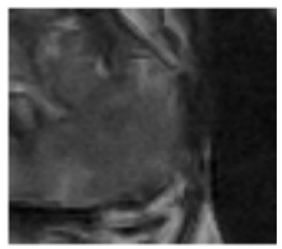

(h) Patient 2 CDSFCRF

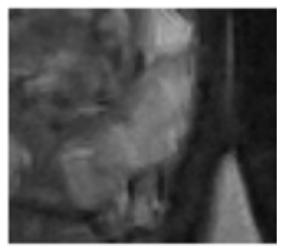

(1) Patient 3 CDSFCRF

Fig. 6 Sample T2w results (zoomed in) for 3 patient images produced using CD-SFCRF, $L_{2}$, and HLo at $32 \%$ sampling ratio. Compared to other methods, CD-SFCRF preserves tissue details and contrast especially in the tumourous regions. The arrow shows tumourous region in the fully sampled image $(\mathbf{a}, \mathbf{e}, \mathbf{i})$

heavily rely on the quality of regions of interests in similar cases in DWI and therefore, it is expected that a reconstructed MRI with better quality will improve the performance. As future work, we will investigate the effect of the proposed compressive sensing method on the detection accuracies of these radiomics-based CAD algorithms with respect to the $L_{2}$ and $\mathrm{H} L_{0}$ methods. Moreover, recently, computational diffusion MRI (CD-MRI) has been introduced which utilizes the wealth of information in DWMRI to computationally construct new sequences of MRI that potentially will help radiologists with more accurate and consistent diagnosis [2,3]. The proposed CD-SFCRF framework will be integrated into CD-MRI algorithms $[2,3]$ to investigate whether CD-SFCRF improves the separability of cancerous and healthy tissues in prostate for these computationally generated MR sequences with respect to the $L_{2}$ and $\mathrm{H} L_{0}$ methods.

The limitations of the proposed CD-SFCRF method that will be addressed in the future direction of this work include the limited sample size. A larger and more diverse dataset will be used to address this limitation. Moreover, in this work, the proposed method was applied only to the prostate. Future work also includes applications of the proposed method to the MRI acquisitions of other organs such as breast or moving organs such as heart. In addition, although the CD-SFCRF can significantly decrease MRI acquisition times, because of the fully-connected nature of this method, the algorithm may require a considerable processing time to complete (although not comparable to original MRI acquisition time). As future work, we will modify the proposed method to improve processing time and the efficiency of the algorithm.

\section{Conclusions}

In this paper, a cross domain stochastic fully connected conditional random field (CD-SFCRF) framework for sparse reconstruction of compressive sensing MRI was presented. The proposed CD-SFCRF framework introduces constraints in both $k$-space and spatial domains within a stochastically fully connected graphical model to produce improved MRI reconstruction. To test the efficacy of the proposed CD-SFCRF framework, quantitative experimentation using peak signal-to-noise ratio (PSNR) analysis was performed on phantom MRI data. Quantitative and qualitative experimentations were also performed on prostate MP-MRI data of 20 patient cases at different sampling rates. The results show an improvement over other tested sparse reconstruction approaches, 


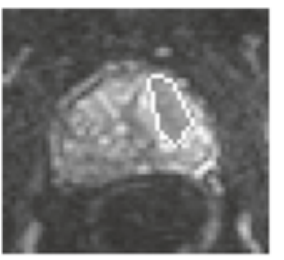

(a) Patient 1 Fully Sampled

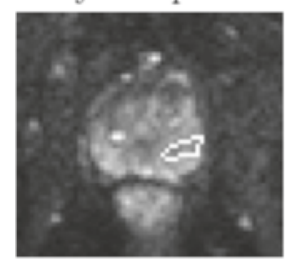

(e) Patient 2 Fully Sampled

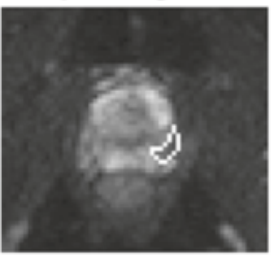

(i) Patient 3

Fully Sampled

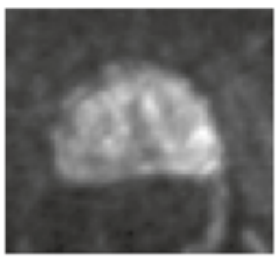

(b) Patient $1 L_{2}$

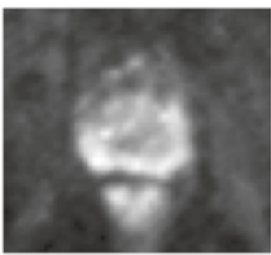

(f) Patient $2 L_{2}$

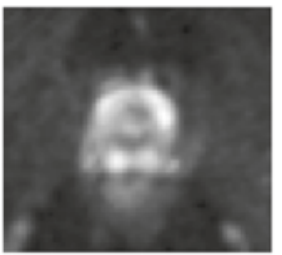

(j) Patient $3 L_{2}$

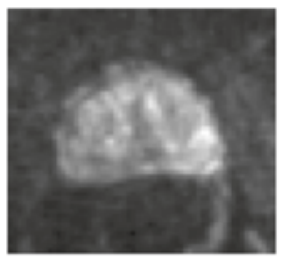

(c) Patient $1 \mathrm{H} L_{0}$

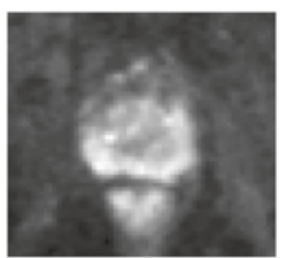

(g) Patient $2 \mathrm{H} L_{0}$

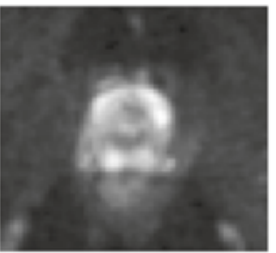

(k) Patient 3 $\mathrm{H} L_{0}$

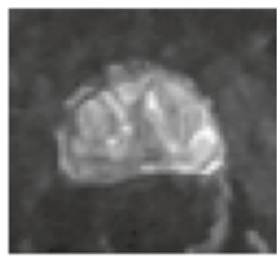

(d) Patient 1 CDSFCRF

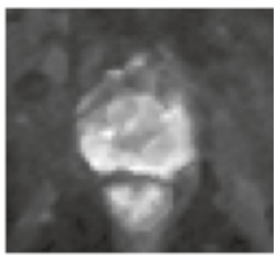

(h) Patient 2 CDSFCRF

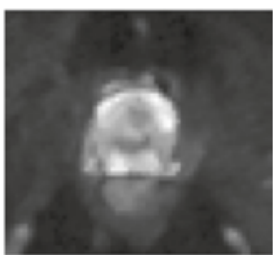

(1) Patient 3 CDSFCRF

Fig. 7 Sample DWI results $\left(b=100 \mathrm{~s} / \mathrm{mm}^{2}\right)$ for three patient cases produced using CD-SFCRF, $L_{2}$, and $H L_{0}$ at $32 \%$ sampling ratio. Compared to other methods, CD-SFCRF preserves tissue details and contrast especially in the tumourous regions. The tumourous region in the fully sampled image is marked $(\mathbf{a}, \mathbf{e}, \mathbf{i})$

especially at low sampling rates. The ability to better utilize available information given very limited information demonstrates the potential of the proposed CDSFCRF framework as a viable reconstruction algorithm for compressive sensing MRI. The proposed CD-SFCRF can significantly reduce MRI acquisition times without sacrificing quality and potential reduction in the accuracy of diagnosis. Reducing MRI acquisition time would reduce related cost significantly and lead to less patient discomfort during the MRI acquisition and more importantly, it would reduce the patient wait times considerably. A fast access to MRI would directly translate to better care given to patients who need it the most.

\section{Appendix 1}

Sparse reconstruction via cross-domain stochastically fully connected conditional random field

The main goal here is to reconstruct image $Y$ given original sparsely sampled $k$-space observations $X$. We model the conditional probability $P(Y \mid X)$ of the full state set $Y$ in spatial domain given the set of sparse measurements $X$ in $k$-space, which can be written as:

$$
P(Y \mid X)=\frac{1}{Z(X)} \exp (-\psi(Y \mid X))
$$

where $Z(X)$ is the normalization function and $\psi($.$) is a$ combination of unary and pairwise potential functions:

$$
\psi(Y \mid X)=\sum_{i=1}^{n} \psi_{u}\left(y_{i}, X\right)+\sum_{\varphi \in C} \psi_{p}\left(y_{\varphi}, X\right)
$$

Here $y_{i} \in Y$ is a single state in the set $Y=\left\{y_{i}\right\}_{i=1}^{n}, y_{\varphi} \in Y$ encodes a clique structure in the set $C$, and $X=\left\{x_{j}\right\}_{j=1}^{n}$ is the observations (radially sub-sampled frequency coefficients) in the frequency domain ( $k$-space). The unary potential $\psi_{u}$ is enforced in the $k$-space while the pairwise potential $\psi_{p}$ is applied in the spatial domain. The unary potential enforces original observations to preserve data fidelity. Since the available observations are captured in $k$ space in MRI, the model must be formulated in a way to be consistent in both $k$-space and spatial domain.

The pairwise potential, on the other hand, has to be in the spatial domain to better preserve image detail since neighboring coefficients in the $k$-space does not contain any meaningful spatial or data consistencies to be utilized by the pairwise potential. Therefore, the optimal way to 


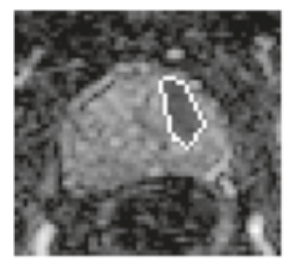

(a) Patient 1 Fully Sampled

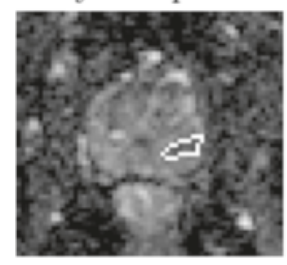

(e) Patient 2

Fully Sampled

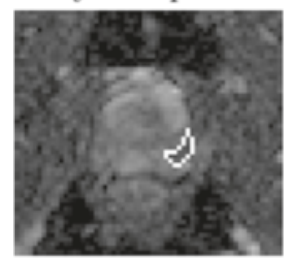

(i) Patient 3

Fully Sampled

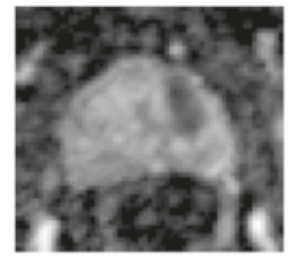

(b) Patient $1 L_{2}$

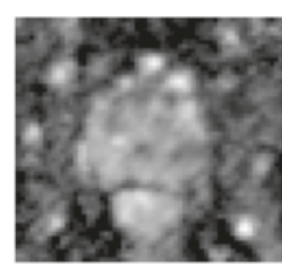

(f) Patient $2 L_{2}$

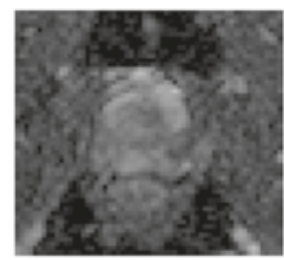

(j) Patient 3 Fully Sampled

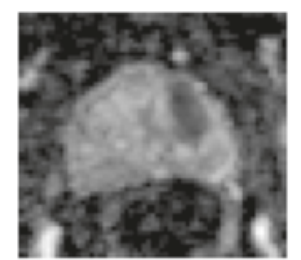

(c) Patient $1 \mathrm{H} L_{0}$

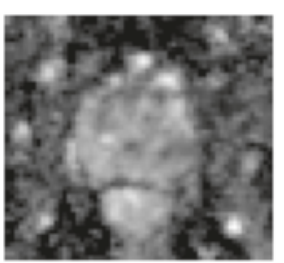

(g) Patient $2 \mathrm{H} L_{0}$

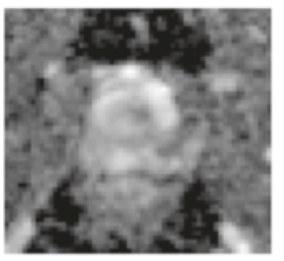

(k) Patient 3 $\mathrm{H} L_{0}$

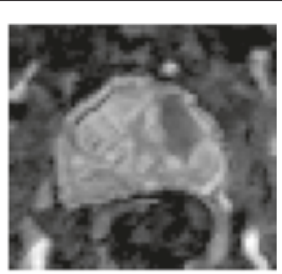

(d) Patient 1 CDSFCRF

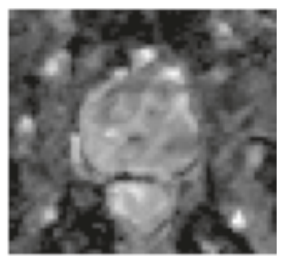

(h) Patient 2 CDSFCRF

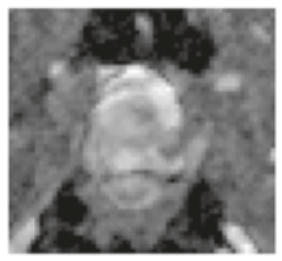

(1) Patient 3 CDSFCRF

Fig. 8 Sample ADC map results for three patient cases produced using CD-SFCRF, $L_{2}$, and $H L_{0}$ at $32 \%$ sampling ratio. Compared to other methods, CD-SFCRF preserves tissue details and contrast especially in the tumourous regions. The tumourous region in the fully sampled image is marked $(\mathbf{a}, \mathbf{e}, \mathbf{i})$

fully utilize available data within this random field model is to formulate the unary potential in the $k$-space and the pairwise potential in the spatial domain.

One of main differences between the proposed CDSFCRF framework from conventional CRF models is to incorporate long-range information in the model and preserve boundaries and image structural properties more effectively which is important here due to sparse available observation. To capture long-range information, CDSFCRF assumes fully connected neighboring structure for the underlying graph which each node $i$ has a set of neighbors

$$
N(i)=\{j \mid j=1: n, j \neq 1\}
$$

where $|N(i)|=n-1$ and includes all other nodes in the graph as neighbors of node $i$. Here the pairwise clique structures are utilized such that:

$$
\begin{aligned}
C & =\left\{C_{p}(i)\right\}_{i=1}^{n} \\
C_{p}(i) & =\left\{(i, j) \mid j \in N(i), 1_{\{i, j\}}^{S}=1\right\} .
\end{aligned}
$$

The active cliques in the inference procedure are determined by the stochastic indicator function $1_{\{i, j\}}^{S}=1$. The indicator function decides whether or not nodes can construct a clique, $C_{p}(i)$ for node $i$. This stochastic indicator function combines spatial and data driven information to model the probability distribution of informative cliques which informative cliques have higher probability to participate in the inference. The set of active cliques are obtained to extract pairwise potentials in Eq. 2 .

As mentioned before, $\psi(\cdot)$ in Eq. 2 is the combination of two potential functions $\psi_{u}($.$) , the unary potential$ and $\psi_{p}($.$) , the pairwise potential. These potential func-$ tions are formulated with their corresponding weights $\lambda$, respectively as:

$$
\begin{aligned}
& \psi_{u}(Y, X)=\sum_{j=1}^{K} \lambda_{j}^{u} F_{j}(Y, X) \\
& \psi_{p}\left(y_{\varphi}, X\right)=\sum_{\left\{y_{i}, y_{j}\right\} \in y_{\varphi}, k=1}^{K^{\prime}} \lambda_{k}^{p} f_{k}\left(y_{i}, y_{j}, X\right)
\end{aligned}
$$

where $\lambda$ controls the importance of each feature function in the energy formulation and it is calculated in the 
training stages. Although it is possible to provide several arbitrary feature functions to model the conditional probability $P(Y \mid X)$, here two feature functions are provided to formulate the image reconstruction for the purpose of sparse reconstruction from compressive sensing MRI. The conditional distribution of $Y$ given $X$ is trained to promote/suppress different features in both the unary and pairwise potentials. Higher $\lambda_{j}^{u}$ values promotes a higher reinforcement of original observations while high $\lambda_{k}^{p}$ values promotes higher consideration of spatial and data driven neighborhood constraints. In Eq. 6, $F$ refers to the frequency domain potential function. The unary potential is calculated in the $k$-space while the pairwise remains in the spatial domain. This is the novelty of the CDSFCRF whihc facilitates for better preservation of fine tissue details and contrast in the reconstructed image. The unary potential function $F_{j}\left(y_{i}, X\right)$ can be formulated as:

$$
F_{j}(Y, X)=\sum_{\omega=-\frac{\pi}{2}}^{\frac{\pi}{2}} \mathscr{F}(Y, \omega)-x_{\omega}
$$

where $\mathscr{F}(\cdot, \cdot)$ is the Fourier operator and returns the $k$ space coefficient corresponding to frequency $\omega$. Based on this formulation, the unary potential is enforced in the $k$-space and in the inferencing step, the model tries to estimate image $Y$ to be consistent to the original $k$-space observation $X=\left\{x_{\omega}\right\}_{\omega=-\frac{\pi}{2}}^{\frac{\pi}{2}}$.

The pairwise function $f_{k}\left(y_{i}, y_{j}, X\right)$ can be formulated as:

$$
f_{k}\left(y_{i}, y_{j}, X\right)=\exp \left(\frac{-\left(y_{i}-y_{j}\right)^{2} \cdot\left(x_{i}-x_{j}\right)^{2}}{3 \sigma^{2}}\right)
$$

where $\sigma$ is a control variable for the amount of weighting node pairs in the clique $\varphi=\{i, j\}$. Contrary to the unary potential, the pairwise potential is enforced in the spatial domain.

\section{Graph representation}

Graph $G(V, E)$ (Fig. 9) is the realization of the CD-SFCRF where $V$ is the set of nodes of the graph representing states $Y=\left\{y_{i}\right\}_{i=1}^{n}, E$ is the set of edges in the graph. Observations $x_{i} \in X$ are made in the $k$-space domain. Our final state estimations $Y$ are in the spatial domain (image). Figure 9 shows the graphical representation of how the spatial and $k$-space domain are incorporated to model the conditional probability $P(Y \mid X) . x_{i}$ comes from sparse measurements in the $k$-space. In the inference procedure, the $k$-space observations are transformed into the spatial domain using the Fourier transform to compute the pairwise potentials. Pairwise potentials are calculated in the spatial domain and transformed into the $k$-space to combine with the unary potential and perform data fidelity. For different types of MRI data, different sparse sampling

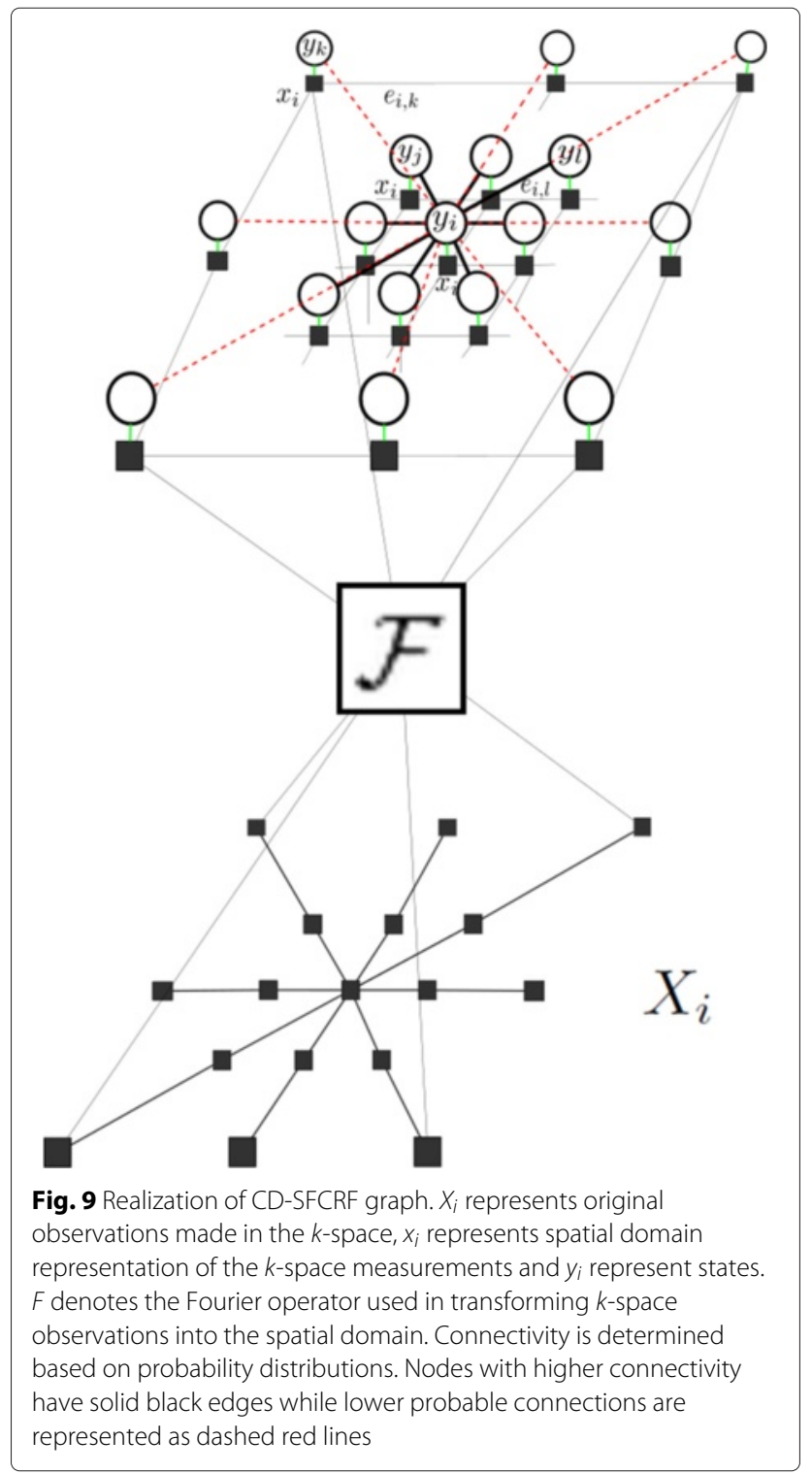

patterns can be used. Furthermore, pairwise connectivity can be trained for specific types of details and tissue structure.

The proposed CD-SFCRF framework utilizes consistencies from the spatial domain through the pairwise potential in conjunction with $k$-space information through the unary potential. A combination of the two potentials is enforced simultaneously. The unary potential utilizes original observations in the $k$-space, while the pairwise potential utilizes the spatial domain representation of the observation/state information and calculates pairwise potentials for nodes in the spatial domain. This allows CD-SFCRF to take advantage of the lower computational complexity introduced by the stochastically fully-connected random field model, while leveraging the original $k$-space observations in improving signal fidelity. 


\section{Funding}

This research has been supported by the Canada Research Chairs programs, Natural Sciences and Engineering Research Council of Canada (NSERC), the Ministry of Research and Innovation of Ontario, and Ontario Institute of Cancer Research (OICR).

\section{Availability of data and materials}

The datasets during and/or analysed during the current study available from the corresponding author on reasonable request pending the approval of the institute.

\section{Authors' contributions}

EL, FK, MJS, and AW contributed to the design and implementation of the concept. EL, FK, MJS, and AW contributed to the design and implementation of the experiments, and performing statistical analysis. FK and MAH were involved in collecting and reviewing the data. All authors contributed to the writing and reviewing of the paper. All authors read and approved the final manuscript.

\section{Competing interests}

The authors declare that they have no competing interests.

\section{Consent for publication}

Not applicable.

\section{Ethics approval and consent to participate}

The institutional research ethics board (Sunnybrook Health Sciences Centre Research Ethics Board) approved this retrospective single institution study and waived the requirement for informed consent.

\section{Author details}

${ }^{1}$ Department of Systems Design Engineering, University of Waterloo, Waterloo, Ontario, Canada. ${ }^{2}$ Department of Medical Imaging, University of Toronto and Sunnybrook Research Institute, Toronto, Ontario, Canada.

\section{Received: 5 April 2016 Accepted: 15 August 2016}

Published online: 26 August 2016

\section{References}

1. Canadian Cancer Statistics Special topic: Predictions of the future burden of cancer in Canada. Technical Report 2015 Canadian Cancer Society www.cancer.ca/statistics.

2. Wong A, Glaister J, Cameron A, Haider MA. Correlated diffusion imaging BMC Med Imaging. 2013;13(1):26. doi:10.1186/1471-2342-13-26.

3. Wong A, Khalvati F, Haider MA. Dual-Stage Correlated Diffusion Imaging. In: IEEE International Symposium on Biomedical Imaging (ISBI). Brooklyn; 2015. p. 75-8.

4. Khalvati F, Wong A, Haider MA. Enhanced Dual-Stage Correlated Diffusion Imaging. In: International Conference of the IEEE Engineering in Medicine and Biology Society (EMBC). Orlando; 2016. p. 5537-40.

5. Esen T, Turkbey B, Patel A, Futterer J. Multiparametric mri in prostate cancer. BioMed Research International. 2014;2014:296810. doi:10.1155/2014/296810.

6. Donoho DL. Compressed sensing. IEEE Trans Inform Theory. 2006;52(4): 1289-306. doi:10.1109/TIT.2006.871582.

7. Candès EJ. Compressive sampling. Int Congr Math. 2006;3:1433-52.

8. Baraniuk R. Compressive Sensing. IEEE Signal Process Mag. 2007:24:1-9.

9. Lustig M, Donoho D, Pauly JM. Magn Reson Med. 2007:58(6):1182-95. doi:10.1002/mrm.21391

10. Liang D, Liu B, Wang J, Ying L. Accelerating SENSE using compressed sensing. Magn Reson Med. 2009;62(6):1574-84. doi:10.1002/mrm.22161.

11. Ye JC, TakS, Han Y, Park HW. Projection reconstruction MR imaging using FOCUSS,. Magn Reson Med. 2007;57(4):764-5. doi:10.1002/mrm.21202.

12. Wong A, Mishra A, Fieguth P, Clausi DA. Sparse reconstruction of breast MRI using homotopic LO minimization in a regional sparsified domain. IEEE Trans Bio-med Eng. 2013;60(3):743-52. doi:10.1109/TBME.2010.2089456.

13. Qu X, Cao X, Guo D, Hu C, Chen Z. Compressed Sensing MRI with Combined Sparsifying transforms and Smooted LO Norm Minimization.
In: IEEE International Conference on Acoustics, Speech and Signal Processing (ICASSP). Dallas; 2010. p. 626-9.

14. Block KT, Uecker M, Frahm J. Magn Reson Med. 2007;57(6):1086-98. doi:10.1002/mrm.21236.

15. Trzasko J, Manduca A. Highly undersampled magnetic resonance image reconstruction via homotopic-minimization. Med Imaging IEEE Trans. 2009;28(1):106-21.

16. Chartrand R. Fast algorithms for nonconvex compressive sensing: Mri reconstruction from very few data. In: Biomedical Imaging: From Nano to Macro, 2009. ISBI'09. IEEE International Symposium On. Boston: IEEE; 2009. p. 262-5.

17. Yin W, Osher S, Goldfarb D, Darbon J. Bregman iterative algorithms for \ell_1-minimization with applications to compressed sensing. SIAM J Imaging Sci. 2008;1 (1):143-68.

18. Figueiredo MA, Nowak RD, Wright SJ. Gradient projection for sparse reconstruction: Application to compressed sensing and other inverse problems. Selected Topics Signal Process IEEE J. 2007;1 (4):586-97.

19. Goldstein $T$, Osher $S$. The split bregman method for 11 -regularized problems. SIAM J Imaging Sci. 2009;2(2):323-43.

20. Wang $Y$, Yang J, Yin W, Zhang Y. A new alternating minimization algorithm for total variation image reconstruction. SIAM J Imaging Sci. 2008;1(3):248-72.

21. Osher $\mathrm{S}$, Burger $\mathrm{M}$, Goldfarb D, Xu J, Yin W. An iterative regularization method for total variation-based image restoration. Multiscale Model Simul. 2005:4(2):460-89.

22. Qu X. Compressed sensing MRI based on nonsubsampled contourlet transform. 2008 IEEE International Symposium on IT in Medicine and Education. 2008693-696. doi:10.1109/ITME.2008.4743955.

23. Yu Y, Hong M, Liu F, Wang H, Crozier S. Compressed sensing MRI using Singular Value Decomposition based sparsity basis. Conf Proc Ann Int Conf IEEE Eng Med Biol Soc. 2011;2011(1):5734-7. doi:10.1109/IEMBS.2011.6091419.

24. Aster R, Borchers B, Thurber C. Parameter estimation and inverse problems. Int Geophys. Series, 90: Elsevier; 2005.

25. Held K, Kops ER, Krause BJ, Wells III WM, Kikinis R, Muller-Gartner HW. Markov random field segmentation of brain $\mathrm{mr}$ images. Med Imaging, IEEE Trans. 1997;16(6):878-6.

26. Blake A, Kohli P, Rother C. Markov Random Fields for Vision and Image Processing. Cambridge: MIT Press; 2011.

27. Lafferty J, McCallum A, Pereira FCN. Conditional random fields: Probabilistic models for segmenting and labeling sequence data. In: ICML (International Conference on Machine Learning); 2001. p. 282-9.

28. Nyquist $\mathrm{H}$. Certain topics in telegraph transmission theory. Am Inst Electrical Eng Trans. 1928;47(2):617-44.

29. Shafiee MJ, Wong A, Siva P, Fieguth P. IEEE International Conference on Image Processing (ICIP). Paris; 2014, pp. 4289-93.

30. Ljunggren S. A simple graphical representation of fourier-based imaging methods. J Magn Reson (1969). 1983;54(2):338-43.

31. Khalvati F, Wong A, Haider M. Automated Prostate Cancer Detection via Comprehensive Multi-Parametric Magnetic Resonance Imaging Texture Feature Models. Biomed Central Med Imaging. 201515-27. doi:10.1186/s12880-015-0069-9.

32. Khalvati F, Modhafar A, Cameron A, Wong A, Haider M. A multi-parametric diffusion magnetic resonance imaging texture feature model for prostate cancer analysis. In: MICCAI 2014 Workshop on Computational Diffusion MRI. Boston; 2014. p. 79-88.

33. Cameron A, Khalvati F, Haider M, Wong A. MAPS: A Quantitative Radiomics Approach for Prostate Cancer Detection. IEEE Trans Biomed Eng. 2016;63(6):1145-56.

34. Cameron A, Modhafar A, Khalvati F, Lui D, Shafiee M, Wong A, Haider MA. Multiparametric MRI Prostate Cancer Analysis via a Hybrid Morphological-Textural Model. In: International Conference of the IEEE Engineering in Medicine and Biology Society (EMBC). Chicago; 2014. p. 3357-60.

35. Chung A, Scharfenberger C, Khalvati F, Wong A, Haider M. Textural Distinctiveness in Multi-Parametric Prostate MRI for Suspicious Region Detection. In: International Conference on Image Analysis and Recognition (ICIAR). Ontario; 2015. p. 368-76.

36. Zhang J, Khalvati F, Wong A, Haider M. Superpixel-based Prostate Cancer Detection from Diffusion Magnetic Resonance Imaging. Vision Lett. 2015;1(1):107. 